\title{
Big Data and Intelligent Decisions: Introduction to the Special Issue
}

\author{
Deng-Feng $\mathrm{Li}^{1} \cdot$ Peide $\mathrm{Liu}^{2} \cdot \mathrm{Kevin} \mathrm{W} . \mathrm{Li}^{3}$
}

Published online: 21 October 2021

(c) The Author(s), under exclusive licence to Springer Nature B.V. 2021

Rapid development of information and communication technology, such as the Internet, Internet of things, cloud computing, 5G, artificial intelligence, and blockchain, has radically changed the landscape of many industries and reshaped the research agendas in economics and management. It is now common for different industrial processes and research areas to become increasingly inter-connected, and large amounts of data have been collected and made available. In the face of very large data sets, significant difficulties and challenges often arise in constructing rational and practical models to assist the decision making process. As a result, research paradigms must shift from traditional qualitative and quantitative methods to big data analytics and intelligent decision models such as fuzzy cognition and reasoning, data mining, association analysis, evidence theory, multi-agent modeling, artificial intelligence, intelligent computing, heuristic algorithms, fuzzy clustering analysis, neural network analysis, deep learning, machine learning, deep reinforcement learning, and social network analysis. Recent years have witnessed widespread applications of data-driven decision paradigms and intelligent decision models to different (often multidisciplinary) fields. Especially, in the past several years, researchers have developed a variety of big data and intelligent decision tools to handle challenging problems in business and economics ranging from digital economy to platform operations management, green ecological environment, sustainable development, low-carbon supply chain management, tourism, and transportation.

This special issue of Group Decision and Negotiation entitled "Big Data and Intelligent Decisions" focuses on theoretical and empirical investigations of big data and intelligent decision tools in group decision and negotiation processes. The aim is to present high-quality papers that report novel big data and intelligent group

Deng-Feng Li

lidengfeng@uestc.edu.cn

1 School of Management and Economics, University of Electronic Science and Technology of China, Chengdu 611731, Sichuan, China

2 School of Management Science and Engineering, Shandong University of Finance and Economics, Jinan 250014, Shandong, China

3 Odette School of Business, University of Windsor, Windsor, ON N9B 3P4, Canada 
decision and negotiation models in digital economies, innovation, business and management, social media and advertising, green low-carbon supply chain, tourism and transportation, and sustainable development. Following the peer-review process according to the aims and scope of Group Decision and Negotiation, nine papers are accepted for publication in this special issue. These papers address different topics of big data and intelligent decision methods involving large-scale group decision making (LGDM) and cooperative behavior as briefly summarized and reviewed below.

About the Papers in this Special Issue

Based on the specific research topics, the nine papers are categorized into three groups: data-driven large-scale group decision making and consensus models, intelligent recommendation methods in large-scale group decision making, and assessment methods of multi-criteria group decision making and cooperative games in a hierarchy structure.

\section{Data-driven Large-Scale Group Decision Making and Consensus Models}

A key problem in group decision making is how to aggregate different opinions of individual decision-makers (DMs) to reach group consensus. Traditionally, this was solved by constructing a minimum cost consensus model under the assumption that DMs' unit adjustment costs are symmetric along the upward and downward directions. However, in most real-world applications, these costs are usually asymmetric in the two directions due to the observation that it is easier for DMs to decrease the cost than increase it. The paper "Consensus Modeling with Asymmetric Cost Based on Data-Driven Robust Optimization” (https://doi.org/10.1007/s10726-020-09707w), by Shaojian Qu, Yefan Han, Zhong Wu, and Hassan Raza, focuses on modeling group consensus with asymmetric unit adjustment costs through introducing a data-driven robust optimization method, where the exact distributions of uncertain cost parameters are unknown with only the bounds given a priori. First, four uncertainty sets (the box, ellipsoid, polyhedron, and interval-polyhedron sets) are introduced to describe the uncertain cost parameters. Then, a series of group consensus cost robust optimization models are constructed under asymmetric unit adjustment cost constraints and the aforesaid four uncertainty sets. Finally, data-driven group consensus cost robust optimization models are developed based on the uncertainty set of asymmetric unit adjustment costs calibrated by using the pricing information of the new product MACUBE 550 in the DEEPCOOL company. Sensitivity analysis shows that the proposed group consensus cost robust optimization models are superior to the existing minimum cost consensus models. The proposed framework can also be applied to other areas such as multi-criteria group decision making and blockchain consensus mechanisms.

In the paper "Hierarchical Punishment-Driven Consensus Model for Probabilistic Linguistic Large-Group Decision Making with Application to Global Supplier Selection" (https://doi.org/10.1007/s10726-020-09681-3), Sumin Yu, Zhijiao Du, and Xuanhua $\mathrm{Xu}$ investigate how to implement a consensus reaching process (CRP) 
for LGDM with probabilistic linguistic evaluation information. The authors first define and formulate probabilistic linguistic LGDM. Its main constituent elements are characterized and novel operational laws are proposed to preprocess probabilistic linguistic information. Then, a hierarchical clustering algorithm is employed to divide a large group of 20 or more DMs into several small-scale subgroups or clusters of DMs with similar opinions, which are regarded as the basic units in the group decision making process. Subsequently, three levels of consensus measures and two adjustment strategies are put forward to refine the scope of measures and adjustments to the matrix at the element level. Finally, a hierarchical punishment-driven large-scale group consensus model is established to iteratively guide the adjustments of cluster opinions and alleviate human supervision of the CRP. The key benefit of the developed framework is a proper reduction of opinion distortions with a goal of achieving consensus within a specified number of iterations. This approach effectively overcomes the scalability challenge with a large number of DMs and automatically provides guidance for adjusting DMs' opinions under properly set parameters.

A group CRP has to consider its cost effect since any preference (or opinion) adjustment is associated with a cost (e.g., effort and time), which is typically limited. The paper "Impact of Decision Rules and Non-cooperative Behaviors on Minimum Consensus Cost in Group Decision Making" (https://doi.org/10.1007/s10726020-09653-7), by Weijun Xu, Xin Chen, Yucheng Dong, and Francisco Chiclana, examines how decision rules (e.g., decision weights, aggregation functions, and non-cooperative behaviors) affect consensus cost in a group CRP. First, a theoretical analysis establishes that a minimal-consensus-cost decision rule can be modeled by the ordered weighted average operator associated with the weight vector in which the first and last components are equal to 0.5 while all others are equal to 0 . Then, from an analytical point of view, three simulation experiments are designed to reveal the joint impact of non-cooperative behaviors and decision rules on the minimal consensus cost. It is further confirmed that non-cooperative behavior significantly increases the minimum consensus cost in the group CRP. This study offers new insights on how decision rules and non-cooperative behaviors influence consensus cost in group CRP.

\section{Intelligent Recommendation Methods in Large-Scale Group Decision Making}

In the paper "The Recommendation Method for Hotel Selection Under Traveller Preference Characteristics: A Cloud-Based Multi-Criteria Group Decision Support Model" (https://doi.org/10.1007/s10726-021-09735-0), Xiao-Kang Wang, ShengHui Wang, Hong-Yu Zhang, and Jian-Qiang Wang regard hotel selections as a multicriteria LGDM problem. This decision process presumably has to consider online reviews, including both user ratings and text comments expressing users' (or DMs') opinions on six criteria: location, cleanliness, comfort level, sleep quality, value, and service. Thus, the authors propose a multi-criteria LGDM support framework for hotel recommendations by using the probability linguistic term sets and an unbalanced trapezoidal cloud model. Through text mining, traveler preference and hotel 
characteristics are extracted from a large sample of online reviews about London hotels on a global tourism platform, TripAdvisor.com. The proposed model can effectively overcome the limitations of existing recommendation methods based on tourist groups and traveler types. It is also able to process a large amount of online review data and avoid excessive information loss. This study not only enables travelers to make a more satisfactory hotel selection but also provides a better decision support tool for tourism websites to recommend more appropriate hotels for their users.

Evaluating and screening product ideas can be treated as an LGDM problem involving a large number of experts and DMs with different knowledge structures and educational backgrounds. The aim of the paper "A Dynamic Intelligent Recommendation Method Based on the Analytical ER Rule for Evaluating Product Ideas in Large-Scale Group Decision-Making" (https://doi.org/10.1007/s10726-020-09687$\mathrm{x}$ ) by Yuan-Wei Du and Yu-Kun Shan is to develop a dynamic intelligent recommendation method for evaluating product ideas. This approach starts with constructing a new evaluation criteria system for product ideas with both input and output criteria. Then, a basic probability assignment function is employed to effectively extract the experts' authentic evaluation information and an analytical evidence reasoning rule is utilized to combine both individual and group evaluation information and obtain the final evaluation results. The dynamic component of this method allows for realtime updating, screening, and ranking of product ideas and the intelligent aspect is reflected in the inference mechanism based on the partial assessment information for product ideas furnished by the experts. The proposed approach can then recommend optimal product ideas by inferring from the experts' partial information without impairing its authenticity. The dynamic nature of this method makes it ideal to handle rapid flow of information and knowledge on network platforms, thereby enhancing innovation efficiency by recommending appropriate product ideas.

Voting for talent shows in mass media is formulated as a social network group decision making (SN-GDM) in which data collected from popular SNs are analyzed to estimate the influence strength among members (participants or representatives) in the group CRP. The aim of the paper "A Voting Mechanism Designed for Talent Shows in Mass Media: Weighted Preference of Group Decision Makers in Social Networks Using Fuzzy Measures and Choquet Integral" (https://doi.org/10.1007/ s10726-020-09666-2), by Mei Cai, Li Yan, Zaiwu Gong, and Guo Wei, is to devise an objective and fair voting mechanism for talent shows in mass media that properly reflects public opinions. First, interactions of the representatives and their voting powers are measured by using fuzzy measures, the Choquet integral, and the associated SNs. A weight determination model is constructed to derive the representatives' weights by observing their behaviors in ranking alternatives based on a communication and CRP. Then, through introducing a harmony degree to gauge the difference between the original and revised opinions after considering individual interactions, a multiple-group hierarchy decision model is established to solve SN-GDM problems where the Choquet integral is employed to reduce the impact of synergy and redundancy owing to the interactions among representatives. The proposed voting mechanism for talent shows in mass media furnishes a new perspective on knowledge management and social network decision making. 


\section{Assessment Methods of Multi-criteria Group Decision Making and Cooperative Games in a Hierarchy Structure}

Ordinal assessments of alternatives in multi-criteria group decision making usually take two forms, ordinal scales and ordinal intervals. However, these two forms cannot properly characterize DMs' ordinal preferences in some real-world situations. As such, the paper "Multi-criteria Group Decision Making with Various Ordinal Assessments" (https://doi.org/10.1007/s10726-020-09677-z), by Wenjun Chang, Chao Fu, Nanping Feng, and Shanlin Yang, introduces a new type of ordinal assessments, referred to as an ordinal distribution. This new ordinal characterization differs from the two existing types and can be regarded as a special case of belief distributions in evidential reasoning for ranking alternatives. This paper unifies the three types of ordinal assessments by transforming ordinal scales and ordinal intervals into ordinal distributions. Theoretical analysis is then conducted to verify the internal consistency of the transformation process. Subsequently, based on the unified ordinal distributions, a dominating degree expressing the possibility of one alternative being superior to another is introduced for pairwise comparison of alternatives and ranking them. Finally, a unified method is developed for solving multi-criteria group decision making with the aforesaid three types of ordinal assessments, which has potentials to solve other real-world problems such as control mode selection and component supplier selection.

By analyzing sustainable development indicators and decision making methods for investment in sustainable development enterprises (SDEs), the paper "An Innovative Indicator System and Group Decision Framework for Assessing Sustainable Development Enterprises" (https://doi.org/10.1007/s10726-019-096470), by Xiang Deng, Xiang Cheng, Jing Gu, and Zeshui Xu, frames SDE assessment as a multi-criteria group decision making problem. First, a set of sustainable development indicators (criteria or attributes) is introduced for investors to assess SDEs. The indicators cover three broad categories, environment, social responsibility, and governance, and consist of 14 specific criteria. Then, to measure subjectivity of indicator values and elicit the relative weights of investors in group decision making, an optimization-based consensus model is constructed by combining the q-rung orthopair fuzzy set with multiplicative multi-objective optimization by a ratio analysis method. The proposed method can assist investors in assessing SDEs.

Transferable utility cooperative games, often called cooperative games for short, are mainly concerned with coalition formation of a finite number of players (or DMs) and the rational allocation of payoffs generated from cooperation. Players and coalitions (or unions) of players sometimes organize into bigger coalitions (or unions) to improve their bargaining power in distributing the worth of the grand coalition, resulting in a hierarchy structure (or a level structure) of players. The paper "The Equal Surplus Division Value for Cooperative Games with a Level Structure" (https://doi.org/10.1007/s10726-020-09680-4) by XunFeng $\mathrm{Hu}$ and Deng-Feng Li puts forward three axiomatizations of the level equal surplus division value for cooperative games with a level structure. First, the 
level equal surplus division value for cooperative games with a level structure is defined level by level among unions of the level structure based on the concept of the equal surplus division value. Then, the authors introduce three axioms: population solidarity within unions, level population solidarity, and top reduced game consistency. Finally, three axiomatizations are proposed for the level equal surplus division value for cooperative games with a level structure by properly combining the aforementioned three axioms with variations of other well-known axioms, such as efficiency, standardness, and quotient game property. The authors prove that the iterative value of the ordered tree cooperative games can be viewed as a special case of the level equal surplus division value for cooperative games with a level structure if the former games can be transformed into the latter ones.

Acknowledgements We sincerely thank all authors for their contributions to this special issue and the reviewers for volunteering their time and expertise to review the submissions. We are grateful to the Co-Editors-in-Chief of Group Decision and Negotiation, Professor GJ de Vreede and Professor Mareike Schoop, and especially the late Editor-in-Chief, Professor Gregory E. Kersten, for their great support and guidance throughout the process of organizing this special issue.

Publisher's Note Springer Nature remains neutral with regard to jurisdictional claims in published maps and institutional affiliations. 